\title{
OPTIMAL CONTROL FOR THE DEGENERATE ELLIPTIC LOGISTIC EQUATION
}

\author{
M. Delgado ${ }^{1}$, J. A. Montero ${ }^{2}$ and A. Suárez ${ }^{1}$ \\ 1. Dpto. Ecuaciones Diferenciales y Análisis Numérico \\ Fac. Matemáticas, C/ Tarfia s/n \\ C. P. 41012, Univ. Sevilla, Sevilla, Spain \\ 2. Dpto. Análisis Matemático \\ C. P. 18071, Univ. Granada, Granada, Spain \\ e-mails: delgado@numer.us.es, jmontero@goliat.ugr.es and suarez@numer.us.es
}

\begin{abstract}
We consider the optimal control of the harvesting of the diffusive degenerate elliptic logistic equation. Under certain assumptions, we prove the existence and uniqueness of an optimal control. Moreover, the optimality system and a characterization of the optimal control are also derived. Sub-supersolution method, singular eigenvalue problem and differentiability with respect to the positive cone are the techniques used to get our results.
\end{abstract}

Key Words. Degenerate logistic equation, Singular eigenvalue problems, Optimal control. AMS Classification. Primary 49J20, 49K20, 92D25, Secondary 35J65.

Running head. Optimal control for degenerate logistic equation 


\section{Introduction}

This work considers the optimal harvesting control of a species whose state is governed by the degenerate (nonlinear slow diffusion) elliptic logistic equation, i.e.,

$$
\begin{cases}-\Delta w^{m}=(a-f) w-e w^{2} & \text { in } \Omega \\ w=0 & \text { on } \partial \Omega\end{cases}
$$

where $\Omega$ is a bounded and regular domain of $\mathbb{R}^{N}, N \geq 1 ; m>1 ; a, f$ and $e$ are bounded functions with some restrictions that will be detailed below.

Equ. (1.1) was introduced in populations dynamics by Gurtin and MacCamy in [5] describing the behaviour of a single species inhabiting in $\Omega$ and whose population density is $w(x)$. Since the population is subject to homogeneous Dirichlet boundary conditions, we are assuming that $\Omega$ is fully surrounded by inhospitable areas. In such model, the positive function $e(x)$ describes the limiting effects of crowding in the species and $a(x)$ represents the growth rate of the species. The function $f(x)$ denotes the distribution of control harvesting of the species. Since $f$ will be considered non-negative, observe that $f$ leads by reducting the growth rate. Finally, the operator $-\Delta$ measures the diffusion, i.e., the moving rate of the species from high density regions to low density areas. In this case, $m>1$ (nonlinear slow diffusion) means that the diffusion is slower than in the linear case $m=1$, which gives rise to more realistic biological results, see [5].

To study (1.1), we make the change of variables $w^{m}=u$ and obtain

$$
\begin{cases}-\Delta u=(a-f) u^{\alpha}-e u^{\beta} & \text { in } \Omega \\ u=0 & \text { on } \partial \Omega\end{cases}
$$

with $\alpha=1 / m$ and $\beta=2 / m$. Under hypothesis $(H 2)$ below, we prove that for each $f$, there exists a unique positive solution of (1.2), that it will be denoted by $u_{f}$. The optimal control criteria is to maximize the payoff functional

$$
J(f):=\int_{\Omega}\left(\lambda u_{f} h(f)-k(f)\right)
$$

where $h \in C^{1}\left(\mathbb{R}^{+} ; \mathbb{R}^{+}\right), k \in C^{2}\left(\mathbb{R}^{+} ; \mathbb{R}^{+}\right)$and $\lambda>0$ will be considered as parameter. $J$ represents the difference between economic revenue measured by $\int_{\Omega} \lambda u_{f} h(f)$ and the control cost measured by $\int_{\Omega} k(f)$. Here, $\lambda$ describes the quotient between the price of the species and 
the cost of the control.

The special case (quadratic functional)

$$
h(t)=t \quad \text { and } \quad k(t)=t^{2},
$$

was introduced in dynamics population by Leung and Stojanovic in [10] (see also [3], [9] and references therein).

An optimal control is a function $f \in \mathcal{C}$, where $\mathcal{C}$ is a suitable subset of $L^{\infty}(\Omega)$, such that

$$
J(f)=\sup _{g \in \mathcal{C}} J(g)
$$

In the case $m=1$, i.e., $\alpha=1$ and $\beta=2$, and $h(t)=t$ and $k(t)=t^{2}$, this problem has been studied in detail in [3], [10] and [11]. In fact, some results of this work have been motivated by [3]. In these papers, under certain assumptions in the coefficients of the problem, the authors obtained the existence and uniqueness of the optimal control, as well as a characterization of the optimal control by means the solution of the optimality system. To obtain the results, the authors used mainly the sub-supersolution method, the derivability of the maps $f \mapsto u_{f}$ and $f \mapsto J(f)$ and the expressions of their derivatives.

When $m>1$, i.e. $\alpha<1$, this derivability is rather difficult than in the case $m=1$, because it involves linear elliptic and eigenvalue problems with unbounded potentials in a neighbourhood of $\partial \Omega$. These difficulties have been solvented by using results of singular eigenvalue problems from [2] and [6], and some classical ones of Krasnoselskii, see [7]. They let us deduce the Fréchet derivability from the Gâteaux derivability with respect to the positive cone. Moreover, the introduction of the functions $h$ and $k$ in the payoff functional leads us to establish the hypotheses to assure the existence and uniqueness of the optimal control.

An outline of this work is as follows: in Section 2 we introduce some notations and we give some results of the existence and uniqueness of the principal eigenvalue and of solution of a linear elliptic problems with unbounded potentials. In Section 3 we show the existence and uniqueness of positive solution of (1.2), collecting a result from [4]. Moreover, we study the derivability of the map $f \mapsto u_{f}$ giving an explicit expression of that. In Section 4, we show that for $\lambda$ sufficiently small there exists a unique optimal control. In the last Section we characterize the optimal control. This characterization provides us the optimality system and certain regularity of the optimal control. It is well known that this regularity can suggest numeric methods to approximate the optimal control, which are not considered in this work. 


\section{Preliminaries}

In this paper we use the following notation: $\Omega$ is a bounded domain in $\mathbb{R}^{N}$ with a smooth boundary $\partial \Omega$ and $\gamma \in(0,2)$ fixed. For any $f \in L^{\infty}(\Omega)$ we denote

$$
\begin{gathered}
f_{M}:=\operatorname{ess} \sup f \quad f_{L}:=\operatorname{essinf} f \\
L_{+}^{\infty}(\Omega):=\left\{f \in L^{\infty}(\Omega): f_{L} \geq 0\right\} \quad L_{-}^{\infty}(\Omega):=\left\{f \in L^{\infty}(\Omega): f_{M} \leq 0\right\} .
\end{gathered}
$$

Moreover, we denote by $P$ the non-negative cone of $C_{0}^{1}(\bar{\Omega})$, whose interior is

$$
\operatorname{int}(P):=\left\{u \in C_{0}^{1}(\bar{\Omega}): u>0 \quad \text { in } \Omega, \partial u / \partial n<0 \quad \text { on } \partial \Omega\right\}
$$

where $C_{0}^{1}(\bar{\Omega})=\left\{u \in C^{1}(\bar{\Omega}): u=0 \quad\right.$ on $\left.\partial \Omega\right\}$ and $n$ is the outward unit normal at $\partial \Omega$.

Finally, for any $\Omega^{\prime} \subset \Omega, \sigma_{1}^{\Omega^{\prime}}$ and $\varphi_{1}^{\Omega^{\prime}}$ stand for the principal eigenvalue and the corresponding positive eigenfunction of the operator $-\Delta$ and homogeneous boundary Dirichlet condition with $\left\|\varphi_{1}^{\Omega^{\prime}}\right\|_{\infty}=1$. In particular, we write $\sigma_{1}:=\sigma_{1}^{\Omega}$ and $\varphi_{1}:=\varphi_{1}^{\Omega}$.

Assume

$$
M \in L_{l o c}^{\infty}(\Omega) \quad \text { verifying } \quad M(x) d_{\Omega}(x)^{\gamma} \in L^{\infty}(\Omega),
$$

where $d_{\Omega}(x):=\operatorname{dist}(x, \partial \Omega)$.

Given $\sigma \in \mathbb{R}$ and $f \in L^{\infty}(\Omega)$, we consider the following problems

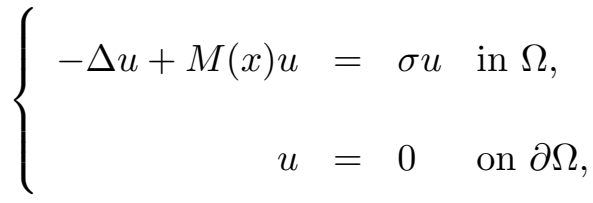

$$
\begin{aligned}
& \left\{\begin{aligned}
-\Delta u+M(x) u & =f \quad \text { in } \Omega \\
u & =0 \quad \text { on } \partial \Omega .
\end{aligned}\right.
\end{aligned}
$$

Remark 2.1 Observe that we are not assuming that $M \in L^{\infty}(\Omega)$ and that a weak solution of (2.2) or an associated eigenfunction to the eigenvalue $\sigma$ of (2.1) are well defined by the Hardy inequality, see for instance [8].

The next result follows from [2] and [6]. We include it for the reader's convenience.

Theorem 2.2 Assume that $M$ satisfies (H1). Then: 
Optimal control for degenerate logistic equation

a) There exists a unique principal eigenvalue (i.e., a real eigenvalue with an associated eigenfunction in int $(P))$, which is simple and we denote it by $\sigma_{1}(-\Delta+M)$. Moreover, it satisfies

$$
\sigma_{1}(-\Delta+M)=\inf _{u \in H_{0}^{1}(\Omega) \backslash\{0\}}\left\{\frac{\int_{\Omega}|\nabla u|^{2}+\int_{\Omega} M(x) u^{2}}{\int_{\Omega} u^{2}}\right\} .
$$

b) (Strong Maximum Principle) $\sigma_{1}(-\Delta+M)>0$ if and only if $v \in W^{2, p}(\Omega) \cap C^{1}(\bar{\Omega})$, with $p>N$ such that $v \neq 0,-\Delta v+M(x) v \geq 0$ in $\Omega, v \geq 0$ on $\partial \Omega$, then $v \in \operatorname{int}(P)$.

By the variational characterization of $\sigma_{1}(-\Delta+M)$, it follows:

Proposition $2.3 \quad$ a) (Monotonicity respect to the potential) Assume that $M_{i}, i=1,2$ satisfy (H1) and $M_{1} \leq M_{2}$. Then

$$
\sigma_{1}\left(-\Delta+M_{1}\right) \leq \sigma_{1}\left(-\Delta+M_{2}\right)
$$

b) (Continuity respect to the potential) Assume that $M_{n}, M, n \in \mathbb{N}$ satisfy (H1) with

$$
\int_{\Omega} M_{n} \varphi^{2} \rightarrow \int_{\Omega} M \varphi^{2}, \quad \text { as } n \rightarrow \infty \text { and for all } \varphi \in H_{0}^{1}(\Omega) \text {. }
$$

Then,

$$
\sigma_{1}\left(-\Delta+M_{n}\right) \rightarrow \sigma_{1}(-\Delta+M) \text { as } n \rightarrow \infty \text {. }
$$

The following estimate will play an important role in the next sections.

Lemma 2.4 Assume that $M_{n}, M, n \in \mathbb{N}$ satisfy $(H 1), \sigma_{1}(-\Delta+M)>0$ and (2.3). Then, there exist a positive constant $C_{0}<1$ (independient of $n$ ) and $n_{0}\left(C_{0}\right) \in \mathbb{N}$ such that

$$
C_{0} \int_{\Omega}|\nabla u|^{2} \leq \int_{\Omega}|\nabla u|^{2}+\int_{\Omega} M_{n} u^{2} \quad \forall u \in H_{0}^{1}(\Omega), \forall n \geq n_{0} .
$$

Proof: Since $\sigma_{1}(-\Delta+K M) \rightarrow \sigma_{1}(-\Delta+M)>0$ as $K \downarrow 1$, there exists $K_{0}>1$ such that $\sigma_{1}\left(-\Delta+K_{0} M\right)>0$. Let $C_{0}$ be such that $K_{0}=1 /\left(1-C_{0}\right)$.

To prove (2.4) it is sufficient to show that $\sigma_{1}\left(-\Delta+K_{0} M_{n}\right) \geq 0$ for $n \geq n_{0}$. But $\sigma_{1}(-\Delta+$ $\left.K_{0} M_{n}\right) \rightarrow \sigma_{1}\left(-\Delta+K_{0} M\right)>0$.

The following result shows that (2.2) possesses a unique solution. 
Theorem 2.5 Assume that $M$ satisfies (H1) and $\sigma_{1}(-\Delta+M)>0$. Then, there exists a unique solution $u \in C^{1, \kappa}(\bar{\Omega})$, for some $\kappa \in(0,1)$, of $(2.2)$. Moreover, there exists a constant $K>0$ (independient of $f$ ) such that

$$
\|u\|_{C^{1, \kappa}(\bar{\Omega})} \leq K\|f\|_{\infty}
$$

Proof: For $v \in C_{0}^{1}(\bar{\Omega})$ we consider the problem

$$
\left\{\begin{aligned}
-\Delta u & =-M(x) v & & \text { in } \Omega \\
u & =0 & & \text { on } \partial \Omega .
\end{aligned}\right.
$$

By Proposition 2.3 in [6], there exists a unique solution $u \in C^{2}(\Omega) \cap C^{1, \kappa}(\bar{\Omega})$, for some $\kappa \in(0,1)$, of (2.6) with

$$
\|u\|_{C^{1, \kappa}(\bar{\Omega})} \leq K_{1}\|v\|_{C^{1}(\bar{\Omega})}
$$

Define $G_{1}: C_{0}^{1}(\bar{\Omega}) \mapsto C_{0}^{1, \kappa}(\bar{\Omega}), v \mapsto G_{1}(v)$ the unique solution of (2.6). We have shown that $G_{1}$ is bounded.

For $h \in L^{\infty}(\Omega)$ we consider the problem

$$
\left\{\begin{aligned}
-\Delta u & =h(x) & & \text { in } \Omega, \\
u & =0 & & \text { on } \partial \Omega .
\end{aligned}\right.
$$

It is well known that fixed $h \in L^{\infty}(\Omega)$, there exists a unique solution $u \in W^{2, p}(\Omega)$ of $(2.7)$ for all $p>1$, and

$$
\|u\|_{C^{1, \kappa}(\bar{\Omega})} \leq K_{1}\|u\|_{W^{2, p}(\Omega)} \leq K_{2}\|h\|_{\infty}
$$

We can define the map $G_{2}: L^{\infty}(\Omega) \mapsto C_{0}^{1, \kappa}(\bar{\Omega}), h \mapsto G_{2}(h)$ the unique solution of $(2.7)$. We have got that $G_{2}$ is bounded.

Now, if we define

$$
H: C_{0}^{1}(\bar{\Omega}) \mapsto C_{0}^{1}(\bar{\Omega}), \quad H(u):=u-G_{1}(u),
$$

denote by $i: C_{0}^{1, \kappa}(\bar{\Omega}) \mapsto C_{0}^{1}(\bar{\Omega})$ the compact imbedding and we pose $G:=H \circ i: C_{0}^{1, \kappa}(\bar{\Omega}) \mapsto$ $C_{0}^{1, \kappa}(\bar{\Omega})$, then we can rewrite $(2.2)$ as

$$
G(u)=G_{2}(f)
$$


being $G$ a compact pertubation of the identity. Since $\sigma_{1}(-\Delta+M)>0, G$ is inyective. The Fredholm's Theorem provides us the existence and uniqueness of solution $u \in C_{0}^{1, \kappa}(\bar{\Omega})$ of $(2.2)$ satisfying (2.5).

The next result is an easy consequence of Theorem $2.2 \mathrm{~b}$ ).

Lemma 2.6 a) Assume that $M$ satisfies $(H 1)$ and $\sigma_{1}(-\Delta+M)>0$. Consider $f_{i} \in L^{\infty}(\Omega)$, $i=1,2$ with $f_{1} \leq f_{2}$ and let $u_{i}, i=1,2$ be the respective solutions of (2.2). Then, $u_{1} \leq u_{2}$.

b) Assume that $M_{i}, i=1,2$ satisfy $(H 1)$ and $M_{1} \leq M_{2}$ with $\sigma_{1}\left(-\Delta+M_{1}\right)>0$. Let $u_{i}$, $i=1,2$ be the respective solutions of (2.2). Then, $u_{2} \leq u_{1}$.

\section{The degenerate logistic equation}

Consider

$$
\left\{\begin{aligned}
-\Delta u & =b u^{\alpha}-e u^{\beta} & & \text { in } \Omega, \\
u & =0 & & \text { on } \partial \Omega,
\end{aligned}\right.
$$

and assume that

$$
0<\alpha<1 \leq \beta, \quad b \in L_{+}^{\infty}(\Omega) \backslash\{0\}, \quad e \in \mathcal{A},
$$

where

$$
\mathcal{A}:=\left\{f \in L^{\infty}(\Omega): f_{L}>0\right\}
$$

The next result has been proved in [4] when $b, e \in C^{\nu}(\bar{\Omega}), \nu \in(0,1)$. The proof is also valid in this case.

Theorem 3.1 Assume (H2). The following assertions are true:

a) There exists a unique strictly positive solution $u_{b}$ of (3.1). Moreover, by elliptic regularity $u_{b} \in W^{2, p}(\Omega), p>1$, and so $u_{b} \in C^{1, \kappa}(\bar{\Omega}) \cap$ int $(P)$, with $0<\kappa \leq 1-N / p$.

b) We have the following a priori bound,

$$
\left\|u_{b}\right\|_{\infty} \leq\left(\frac{b_{M}}{e_{L}}\right)^{1 /(\beta-\alpha)} .
$$


c) If $b_{L}>0$, then there exists $\varepsilon_{0}>0$ such that for all $\varepsilon \leq \varepsilon_{0}$, it holds

$$
\varepsilon \varphi_{1}(x) \leq u_{b}(x) \quad \text { c.p.d. } x \in \Omega
$$

where $\varepsilon_{0}>0$ satisfies

$$
b_{L}-\sigma_{1} \varepsilon^{1-\alpha}-e_{M} \varepsilon^{\beta-\alpha}=0 .
$$

d) If $b_{L}=0$, since $b_{M}>0$ there exists a ball $B:=B\left(x_{0}, r\right)$ such that $b_{L, B}>0$ in $B$, where $b_{L, B}$ is the essential infimum of $b$ in $B$. Hence, $\varepsilon \varphi_{1}^{B} \leq u_{b}$ c.p.d. in $B$ for all $\varepsilon \leq \varepsilon_{1}$ and where $\varepsilon_{1}>0$ satisfies

$$
b_{L, B}-\sigma_{1}^{B} \varepsilon^{1-\alpha}-e_{M, B} \varepsilon^{\beta-\alpha}=0 .
$$

Remark 3.2 By (H2), (3.1) satisfies the strong maximum principle and then there exist two positive constants $k_{1}, k_{2}$ such that

$$
k_{1} d_{\Omega}(x) \leq u_{b}(x) \leq k_{2} d_{\Omega}(x) \quad \forall x \in \Omega
$$

The following result plays an important role along the work.

Theorem 3.3 Assume (H2). Then, the map $b \in \mathcal{A} \subset L^{\infty}(\Omega) \mapsto u_{b} \in \operatorname{int}(P) \subset C_{0}^{1}(\bar{\Omega})$ is increasing, continuous and $C^{1}$.

For the proof of this result we use the following elementary lemma.

Lemma 3.4 a) Let $\alpha \in(0,1]$ and $0<t_{1}<t_{2}$ be. Then

$$
\alpha t_{2}^{\alpha-1}\left(t_{2}-t_{1}\right) \leq t_{2}^{\alpha}-t_{1}^{\alpha} \leq \alpha t_{1}^{\alpha-1}\left(t_{2}-t_{1}\right)
$$

b) Let $\beta \in[1,+\infty)$ and $0 \leq t_{1}<t_{2}$ be. Then

$$
\beta t_{1}^{\beta-1}\left(t_{2}-t_{1}\right) \leq t_{2}^{\beta}-t_{1}^{\beta} \leq \beta t_{2}^{\beta-1}\left(t_{2}-t_{1}\right)
$$

Proof of Theorem 3.3: It follows easily that the map is increasing. For the continuity, let $b_{n}, b \in \mathcal{A}$ be such that $b_{n} \rightarrow b$ in $L^{\infty}$, then $\left(b_{n}\right)_{M} \rightarrow b_{M}$. Hence, fixed $\delta>0$ there exists $n_{0} \in \mathbb{N}$ such that for $n \geq n_{0}$

$$
\left.\left\|u_{b_{n}}\right\|_{\infty} \leq\left(\frac{\left(b_{n}\right)_{M}}{e_{L}}\right)^{1 /(\beta-\alpha)} \leq\left(\frac{b_{M}+\delta}{e_{L}}\right)^{1 /(\beta-\alpha)}=C \quad \text { (independient of } n\right),
$$

and so, the sequence $\left\{u_{b_{n}}\right\}$ is bounded in $W^{2, p}(\Omega), p>1$. There exists a subsequence, relabeled by $n$, such that $u_{b_{n}} \rightarrow u$ in $C^{1, \kappa}(\bar{\Omega}), \kappa<1-N / p$. Moreover, $u$ is a weak solution of (3.1). It 
remains to prove that $u=u_{b}$. By the uniqueness of positive solution of (3.1), it suffices to prove that $u>0$. Since $b_{M}>0$, there exist $x_{0} \in \Omega, r_{0}>0$, such that $\left(b_{n}\right) \geq\left(b_{n}\right)_{L, B}>0$ c.p.d. in $B=B\left(x_{0}, r_{0}\right)$, for $n \geq n_{0}$. By Theorem $\left.3.1 \mathrm{~d}\right)$, we have that there exist $\varepsilon_{n}>0$ such that $\varepsilon_{n} \varphi_{1}^{B} \leq u_{b_{n}}$ c.p.d. in $B$ where $\varepsilon_{n}$ is such that

$$
\left(b_{n}\right)_{L, B}-\sigma_{1}^{B} \varepsilon_{n}^{1-\alpha}-e_{M, B} \varepsilon_{n}^{\beta-\alpha}=0 .
$$

Since $\left(b_{n}\right)_{L, B} \rightarrow b_{L, B}$, it follows that $\varepsilon_{n} \rightarrow \varepsilon>0$ where $\varepsilon$ is such that

$$
b_{L, B}-\sigma_{1}^{B} \varepsilon^{1-\alpha}-e_{M, B} \varepsilon^{\beta-\alpha}=0
$$

and so $\varepsilon \varphi_{1}^{B} \leq u$ c.p.d. in $B$ and then $u>0$.

For the derivability we use the Implicit Function Theorem. Fixed $p>N$, we define the map $\mathcal{F}: \mathcal{A} \times \mathcal{U} \subset L^{\infty}(\Omega) \times C_{0}^{1}(\bar{\Omega}) \mapsto L^{p}(\Omega)$ where $\mathcal{U}:=W^{2, p}(\Omega) \cap \operatorname{int}(P)$, as

$$
\mathcal{F}(b, u):=-\Delta u-b u^{\alpha}+e u^{\beta}
$$

$\mathcal{A}$ is an open set in $L^{\infty}(\Omega)$ and it is well known, see [1], that $\mathcal{U}$ is also open in $C_{0}^{1}(\bar{\Omega})$. It is clear that $\mathcal{F}\left(b_{0}, u_{b_{0}}\right)=0$.

We show that $\mathcal{F}$ is $C^{1}$, for which it is sufficient to show it for the second component. We calculate the Gâteaux derivative respect to this, which will be denoted by $D_{G} \mathcal{F}$. Let $(b, u) \in \mathcal{A} \times \mathcal{U}$ and $\xi \in C_{0}^{1}(\bar{\Omega})$ be, then

$D_{G} \mathcal{F}(b, u) \xi:=\lim _{\varepsilon \rightarrow 0} \frac{\mathcal{F}(b, u+\varepsilon \xi)-\mathcal{F}(b, u)}{\varepsilon}=-\Delta \xi-b \lim _{\varepsilon \rightarrow 0} \frac{(u+\varepsilon \xi)^{\alpha}-u^{\alpha}}{\varepsilon}+e \lim _{\varepsilon \rightarrow 0} \frac{(u+\varepsilon \xi)^{\beta}-u^{\beta}}{\varepsilon}$.

We claim that:

$$
\frac{(u+\varepsilon \xi)^{\beta}-u^{\beta}}{\varepsilon} \rightarrow \beta u^{\beta-1} \xi \quad \text { and } \quad \frac{(u+\varepsilon \xi)^{\alpha}-u^{\alpha}}{\varepsilon} \rightarrow \alpha u^{\alpha-1} \xi \quad \text { in } L^{p}(\Omega) \text { as } \varepsilon \rightarrow 0 .
$$

Assume $\varepsilon \downarrow 0$. Using Lemma 3.4, to prove (3.4) it is sufficient to show that

$$
(u+\varepsilon \xi)^{\beta-1} \xi \rightarrow u^{\beta-1} \xi \quad \text { and } \quad(u+\varepsilon \xi)^{\alpha-1} \xi \rightarrow u^{\alpha-1} \xi \quad \text { in } L^{p}(\Omega) \text { as } \varepsilon \downarrow 0 .
$$

The first one is true because $\beta \geq 1$. For the second one, we have

$$
\left\|\left[(u+\varepsilon \xi)^{\alpha-1}-u^{\alpha-1}\right] \xi\right\|_{p}=\left\|\left[(u+\varepsilon \xi)^{\alpha}-(u+\varepsilon \xi) u^{\alpha-1}\right]\left(\frac{\xi}{u+\varepsilon \xi}\right)\right\|_{p} .
$$

Since $u \in \operatorname{int}(P)$, there exist $\varepsilon_{0}>0$ and $k(\varepsilon)$ such that $u+\varepsilon \xi \in \operatorname{int}(P)$ for $\varepsilon \leq \varepsilon_{0}$ and

$$
k(\varepsilon):=\inf _{x \in \bar{\Omega}} \frac{u(x)+\varepsilon \xi(x)}{d_{\Omega}(x)}>0 .
$$


Clearly $k_{0}:=\min \left\{k(0), k\left(\varepsilon_{0}\right)\right\}$ (independent of $\varepsilon$ ) verifies

$$
k_{0} d_{\Omega}(x) \leq u+\varepsilon \xi
$$

On the other hand, since $\xi \in C_{0}^{1}(\bar{\Omega})$, it follows that from the Mean Value Theorem that

$$
|\xi(x)| \leq d_{\Omega}(x)\|\xi\|_{C^{1}(\bar{\Omega})} \quad \text { for } x \in \Omega
$$

Then, using (3.6) and (3.7), we get

$$
\frac{|\xi|}{u+\varepsilon \xi} \leq \frac{|\xi|}{k_{0} d_{\Omega}(x)} \leq C\|\xi\|_{C^{1}(\bar{\Omega})} .
$$

Moreover, by (3.3) and (3.7)

$$
(u+\varepsilon \xi) u^{\alpha-1} \leq(u+\varepsilon \xi) k_{1}^{\alpha-1} d_{\Omega}(x)^{\alpha-1} \leq K d_{\Omega}(x)^{\alpha} \in L^{\infty}(\Omega),
$$

and so,

$$
(u+\varepsilon \xi)^{\alpha}-(u+\varepsilon \xi) u^{\alpha-1} \in L^{\infty}(\Omega) .
$$

Therefore, from (3.5) and (3.8), it follows that

$$
\left\|\left[(u+\varepsilon \xi)^{\alpha-1}-u^{\alpha-1}\right] \xi\right\|_{p} \leq C\left\|(u+\varepsilon \xi)^{\alpha}-(u+\varepsilon \xi) u^{\alpha-1}\right\|_{p}\|\xi\|_{C^{1}(\bar{\Omega})} \rightarrow 0 \quad \text { as } \varepsilon \downarrow 0 .
$$

This proves (3.4), and so that the linear and continuous map

$$
D_{G} \mathcal{F}(b, u) \xi=-\Delta \xi-\alpha b u^{\alpha-1} \xi+\beta e u^{\beta-1} \xi, \quad \forall \xi \in C_{0}^{1}(\bar{\Omega})
$$

is the Gâteaux derivative.

For the continuity of this map, we have to prove that if $\left(b_{n}, u_{n}\right) \rightarrow(b, u)$, then

$$
\left\|D_{G} \mathcal{F}\left(b_{n}, u_{n}\right)-D_{G} \mathcal{F}(b, u)\right\|_{\mathcal{L}\left(C_{0}^{1}(\bar{\Omega}) ; L^{p}(\Omega)\right)} \rightarrow 0
$$

for which, thanks to $\beta \geq 1$, it is sufficient to show that

$$
\sup _{\|\xi\|_{C^{1}(\bar{\Omega})}=1}\left\{\left\|\left(b_{n} u_{n}^{\alpha-1}-b u^{\alpha-1}\right) \xi\right\|_{p}\right\} \rightarrow 0 .
$$

Firstly, observe that

$$
\left(b_{n} u_{n}^{\alpha-1}-b u^{\alpha-1}\right) \xi=\left(b_{n}-b\right) u_{n}^{\alpha-1} \xi+b\left(u_{n}^{\alpha-1}-u^{\alpha-1}\right) \xi .
$$

Since $u_{n} \in \operatorname{int}(P)$, it is well-defined

$$
0<k_{n}:=\inf _{x \in \bar{\Omega}} \frac{u_{n}(x)}{d_{\Omega}(x)} .
$$


By the continuty of the infimum, it follows that

$$
k_{n} \rightarrow k_{0}:=\inf _{x \in \bar{\Omega}} \frac{u(x)}{d_{\Omega}(x)}>0
$$

since $u \in \operatorname{int}(P)$.

Hence, using (3.9) and (3.7), as $n \rightarrow \infty$ we get

$$
\begin{aligned}
\left\|\left(b_{n} u_{n}^{\alpha-1}-b u^{\alpha-1}\right) \xi\right\|_{p} & \leq\left\|b_{n}-b\right\|_{\infty}\left\|u_{n}^{\alpha-1} \xi\right\|_{\infty}+\|b\|_{\infty}\left\|\left(u_{n}^{\alpha-1}-u^{\alpha-1}\right) \xi\right\|_{\infty} \\
& \leq\left\|b_{n}-b\right\|_{\infty} k_{n}^{\alpha-1} d_{\Omega}^{\alpha}\|\xi\|_{C^{1}(\bar{\Omega})}+\|b\|_{\infty}\left\|\left(u_{n}^{\alpha}-u_{n} u^{\alpha-1}\right) \frac{\xi}{u_{n}}\right\|_{\infty} \\
& \leq\|\xi\|_{C^{1}(\bar{\Omega})}\left(\left\|b_{n}-b\right\|_{\infty} k_{n}^{\alpha-1} d_{\Omega}^{\alpha}+\|b\|_{\infty}\left\|u_{n}^{\alpha}-u_{n} u^{\alpha-1}\right\|_{\infty} k_{n}^{-1}\right) \rightarrow 0 .
\end{aligned}
$$

Therefore, $\mathcal{F}$ is $C^{1}$ respect to the second component and the Gâteaux derivative coincides with the Fréchet one. Denote it by $D_{2} \mathcal{F}$.

Finally, we will prove that $D_{2} \mathcal{F}\left(b_{0}, u_{b_{0}}\right)$ is non singular showing that

$$
\sigma_{1}\left(-\Delta-\alpha b_{0} u_{b_{0}}^{\alpha-1}+\beta e u_{b_{0}}^{\beta-1}\right)>0
$$

Indeed, define

$$
M_{b}:=-\alpha b u_{b}^{\alpha-1}+\beta e u_{b}^{\beta-1}
$$

We will prove that $M_{b_{0}}$ satisfies (H1) and $\sigma_{1}\left(-\Delta+M_{b_{0}}\right)>0$. Observe that $M_{b_{0}} \in L_{l o c}^{\infty}(\Omega)$ and that by (3.3), there exists $k_{1}>0$ such that $k_{1} d_{\Omega}(x) \leq u_{b_{0}}$. Then,

$$
\left|M_{b_{0}}(x)\right| d_{\Omega}(x)=\left|-\alpha b_{0}+\beta e(x) u_{b_{0}}^{\beta-\alpha}\right| u_{b_{0}}^{\alpha-1} d_{\Omega}(x) \leq C u_{b_{0}}^{\alpha-1} d_{\Omega}(x) \leq C k_{1}^{\alpha-1} d_{\Omega}(x)^{\alpha-1} d_{\Omega}(x),
$$

i.e., $M_{b_{0}}(x) d_{\Omega}(x)$ is bounded.

On the other hand, since $u_{b_{0}}$ is solution of (3.1) we get that $\sigma_{1}\left(-\Delta-b_{0} u_{b_{0}}^{\alpha-1}+e u_{b_{0}}^{\beta-1}\right)=0$, and so by (H2) and Proposition 2.3 it follows that

$$
\sigma_{1}\left(-\Delta-\alpha b_{0} u_{b_{0}}^{\alpha-1}+\beta e u_{b_{0}}^{\beta-1}\right)>\sigma_{1}\left(-\Delta-b_{0} u_{b_{0}}^{\alpha-1}+e u_{b_{0}}^{\beta-1}\right)=0 .
$$

This proves (3.10). Now, the Implicit Function Theorem assures that there exist two open neighbourhoods $\mathcal{N}$, of $b_{0}$ in $L^{\infty}(\Omega)$ and $\mathcal{M}$, of $u_{b_{0}}$ in $C_{0}^{1}(\bar{\Omega})$, and a $C^{1}$ map $\Phi: \mathcal{N} \mapsto \mathcal{M}$ such that

a) $\Phi\left(b_{0}\right)=u_{b_{0}}$, 
b) $\mathcal{F}(s, \Phi(s))=0$ for any $s \in \mathcal{N}$,

c) $\mathcal{F}(s, y)=0$ with $s \in \mathcal{N}, y \in \mathcal{M}$, then $y=\Phi(s)$.

Since for $s$ near $b$, the equation possesses a unique solution, then $\Phi(s)=u_{s}$. Therefore, $b \mapsto u_{b}$ is $C^{1}$ and the proof is complete.

Along this work, we need the Gâteaux derivative of the map $b \in L_{+}^{\infty}(\Omega) \backslash\{0\} \mapsto u_{b} \in \operatorname{int}(P)$.

Lemma 3.5 Let $b \in L_{+}^{\infty}(\Omega) \backslash\{0\}, g \in L_{+}^{\infty}(\Omega)$ or $g \in L_{-}^{\infty}(\Omega)$, and $\varepsilon \simeq 0$ be such that $b+\varepsilon g \in$ $L_{+}^{\infty}(\Omega) \backslash\{0\}$. Then,

$$
\frac{u_{b+\varepsilon g}-u_{b}}{\varepsilon} \rightarrow \xi_{b, g} \quad \text { in } H_{0}^{1}(\Omega) \text { as } \varepsilon \rightarrow 0,
$$

where $\xi_{b, g}$ is the unique solution of

$$
\left\{\begin{array}{rll}
-\Delta \xi+M_{b}(x) \xi & =g u_{b}^{\alpha} & \text { in } \Omega \\
\xi & =0 & \text { on } \partial \Omega
\end{array}\right.
$$

and $M_{b}$ is defined in (3.11).

Remark 3.6 Since $M_{b}$ satisfies (H1) and by (3.10), it follows from Theorem 2.5 the existence and uniqueness of $\xi_{b, g} \in C_{0}^{1}(\bar{\Omega})$.

Proof: Let $g \in L_{+}^{\infty}(\Omega), \varepsilon>0$ be and define

$$
\xi_{\varepsilon}:=\frac{u_{b+\varepsilon g}-u_{b}}{\varepsilon}
$$

It is easy to show that $\xi_{\varepsilon}$ satisfies

$$
\left\{\begin{aligned}
-\Delta \xi_{\varepsilon}+\left(-b A_{\varepsilon}+e B_{\varepsilon}\right) \xi_{\varepsilon} & =g u_{b+\varepsilon g}^{\alpha} & & \text { in } \Omega \\
\xi_{\varepsilon} & =0 & & \text { on } \partial \Omega
\end{aligned}\right.
$$

where

$$
A_{\varepsilon}(x):=\frac{u_{b+\varepsilon g}^{\alpha}(x)-u_{b}^{\alpha}(x)}{u_{b+\varepsilon g}(x)-u_{b}(x)} \quad B_{\varepsilon}(x):=\frac{u_{b+\varepsilon g}^{\beta}(x)-u_{b}^{\beta}(x)}{u_{b+\varepsilon g}(x)-u_{b}(x)}
$$

Since $b+\varepsilon g>b$ and by the monotony of the map $b \mapsto u_{b}$, it follows that $A_{\varepsilon}, B_{\varepsilon} \in C^{1}(\Omega)$. In fact, $\alpha u_{b+\varepsilon g}^{\alpha-1} \leq A_{\varepsilon} \leq \alpha u_{b}^{\alpha-1}$ and $\beta u_{b}^{\beta-1} \leq B_{\varepsilon} \leq \beta u_{b+\varepsilon g}^{\beta-1} \in L^{\infty}(\Omega)$ and so,

$$
B_{\varepsilon} \rightarrow \beta u_{b}^{\beta-1} \quad \text { as } \varepsilon \downarrow 0
$$


and $\left|A_{\varepsilon}\right| d_{\Omega} \in L^{\infty}(\Omega)$. So, $\sigma_{1}\left(-\Delta-b A_{\varepsilon}+e B_{\varepsilon}\right)$ is well defined. Moreover,

$$
-b A_{\varepsilon}+e B_{\varepsilon} \geq-\alpha b u_{b}^{\alpha-1}+\beta e u_{b}^{\beta-1}
$$

and by a similar reasoning to used in (3.4), we have that as $\varepsilon \downarrow 0$

$$
\int_{\Omega} A_{\varepsilon} \varphi^{2} \rightarrow \alpha \int_{\Omega} u_{b}^{\alpha-1} \varphi^{2}, \quad \int_{\Omega} B_{\varepsilon} \varphi^{2} \rightarrow \beta \int_{\Omega} u_{b}^{\beta-1} \varphi^{2}, \quad \forall \varphi \in H_{0}^{1}(\Omega) .
$$

Hence, by Proposition 2.3, we get

$$
\sigma_{1}\left(-\Delta-b A_{\varepsilon}+e B_{\varepsilon}\right) \rightarrow \sigma_{1}\left(-\Delta-\alpha b u_{b}^{\alpha-1}+\beta e u_{b}^{\beta-1}\right) \quad \text { as } \varepsilon \downarrow 0 .
$$

and by (3.10) and (3.13),

$$
\sigma_{1}\left(-\Delta-b A_{\varepsilon}+e B_{\varepsilon}\right) \geq \sigma_{1}\left(-\Delta-\alpha b u_{b}^{\alpha-1}+\beta e u_{b}^{\beta-1}\right)>0
$$

Then, applying Lemma 2.4, there exists a constant $C$ (independient of $\varepsilon$ ) such that

$C \int_{\Omega}\left|\nabla \xi_{\varepsilon}\right|^{2} \leq \int_{\Omega}\left|\nabla \xi_{\varepsilon}\right|^{2}+\int_{\Omega}\left(-\alpha b u_{b}^{\alpha-1}+\beta e u_{b}^{\beta-1}\right) \xi_{\varepsilon}^{2} \leq \int_{\Omega}\left|\nabla \xi_{\varepsilon}\right|^{2}+\int_{\Omega}\left(-b A_{\varepsilon}+e B_{\varepsilon}\right) \xi_{\varepsilon}^{2}=\int_{\Omega} g u_{b+\varepsilon g}^{\alpha} \xi_{\varepsilon}$ and so, using (3.2), we obtain

$$
\left.\left\|\xi_{\varepsilon}\right\|_{H_{0}^{1}(\Omega)} \leq C \quad \text { (independient of } \varepsilon\right)
$$

Then, of each bounded sequence considered, there exists a weakly convergent sub-sequence. It is not hard to prove that the limit verifies (3.12), and by the uniqueness of solution it follows that $\xi_{\varepsilon} \rightarrow \xi_{b, g}$ in $H_{0}^{1}(\Omega)$.

In the case $g \in L_{-}^{\infty}(\Omega), \varepsilon>0$, it holds $\alpha u_{b}^{\alpha-1} \leq A_{\varepsilon} \leq \alpha u_{b+\varepsilon g}^{\alpha-1}$ and $\beta u_{b}^{\beta-1} \geq B_{\varepsilon} \geq \beta u_{b+\varepsilon g}^{\beta-1}$, and and so, instead of (3.13), we have

$$
-b A_{\varepsilon}+e B_{\varepsilon} \geq-\alpha b u_{b+\varepsilon g}^{\alpha-1}+\beta e u_{b+\varepsilon g}^{\beta-1}
$$

As $\varepsilon \downarrow 0$, we have

$$
\int_{\Omega}\left(-\alpha b u_{b+\varepsilon g}^{\alpha-1}+\beta e u_{b+\varepsilon g}^{\beta-1}\right) \varphi^{2} \rightarrow \int_{\Omega}\left(-\alpha b u_{b}^{\alpha-1}+\beta e u_{b}^{\beta-1}\right) \varphi^{2} \quad \forall \varphi \in H_{0}^{1}(\Omega) .
$$

By Proposition 2.3, we get

$$
\sigma_{1}\left(-\Delta-b A_{\varepsilon}+e B_{\varepsilon}\right) \geq \sigma_{1}\left(-\Delta-\alpha b u_{b+\varepsilon g}^{\alpha-1}+\beta e u_{b+\varepsilon g}^{\beta-1}\right) \rightarrow \sigma_{1}\left(-\Delta-\alpha b u_{b}^{\alpha-1}+\beta e u_{b}^{\beta-1}\right)>0 .
$$

Again, applying Lemma 2.4 we obtain the result. 


\section{Existence and uniqueness of optimal control}

Consider $a$ such that

$$
a \in \mathcal{A} \text {. }
$$

We define the set

$$
\mathcal{C}:=\left\{f \in L_{+}^{\infty}(\Omega): f \leq a\right\} .
$$

When $f \in \mathcal{C}$, we have proved in the previous Section that there exists a unique positive solution of (3.1) with $b=a-f$, and it will denote by $u_{f}$ (if $f=a$, then $u_{f}:=0$.)

For $\lambda>0$ we consider the functional $J: \mathcal{C} \mapsto \mathbb{R}$

$$
J(g):=\int_{\Omega}\left(\lambda h(g) u_{g}-k(g)\right)
$$

where $h \in C^{1}\left(\mathbb{R}^{+} ; \mathbb{R}^{+}\right), h^{\prime}$ is Lipschitz continuous function and $h(s)=0$ if and only if $s=0$; $k \in C^{2}\left(\mathbb{R}^{+} ; \mathbb{R}^{+}\right)$is a convex function and there exists $C>0$ such that $|k(s)| \leq C s^{2}$ and $k^{\prime \prime}(s) \geq k_{0}>0$. We assume:

$$
\lim _{t \rightarrow 0} \frac{k(t)}{h(t)}=0
$$

$$
\lim _{t \rightarrow+\infty} \frac{k(t)}{h(t)}=+\infty, \quad t \mapsto \frac{h(t)}{t} \quad \text { is non-increasing, } \quad t \mapsto \frac{k(t)}{t} \quad \text { is increasing. }
$$

In this Section we want to prove the existence and uniqueness of the optimal control under suitable assumptions. The following result gives us the existence of optimal control.

Theorem 4.1 Assume $(H 3)-(H 4)$. There exists an optimal control, i.e., $f \in \mathcal{C}$ such that

$$
J(f)=\sup _{g \in \mathcal{C}} J(g)
$$

Moreover, the benefit is positive, i.e., $\sup _{g \in \mathcal{C}} J(g)>0$.

Proof: By (3.2), it follows that

$$
\sup _{g \in \mathcal{C}} J(g)<+\infty
$$

and so, there exists a maximizing sequence $f_{n} \in \mathcal{C}$. Then, there exists a subsequence, relabeled by $f_{n}$, such that

$$
f_{n} \rightarrow f \in \mathcal{C} \quad \text { in } L^{2}(\Omega) \text { and } \quad u_{f_{n}} \rightarrow u_{f} \quad \text { in } H_{0}^{1}(\Omega)
$$


and by the regularity of $h$,

$$
h\left(f_{n}\right) \rightarrow h(f) \in \mathcal{C} \quad \text { in } L^{2}(\Omega),
$$

and then,

$$
\int_{\Omega} h\left(f_{n}\right) u_{f_{n}} \rightarrow \int_{\Omega} h(f) u_{f}
$$

By the hypothesis on $k$, the map $\Phi: L^{2}(\Omega) \mapsto \mathbb{R}$ defined by

$$
\Phi(g):=\int_{\Omega} k(g)
$$

is continuous (see Lemma 17.1 in [12], for instance) and convex, and so w.l.s.c. Then,

$$
\int_{\Omega} k(f) \leq \underline{\lim }_{n \rightarrow \infty} \int_{\Omega} k\left(f_{n}\right)
$$

Hence,

$$
J(f)=\int_{\Omega} \lambda h(f) u_{f}-k(f) \geq \varlimsup_{n \rightarrow \infty} \int_{\Omega} \lambda h\left(f_{n}\right) u_{f_{n}}-k\left(f_{n}\right)=\sup _{g \in \mathcal{C}} \int_{\Omega} \lambda h(g) u_{g}-k(g) .
$$

Finally, we take $f=\varepsilon>0$, then

$$
J(\varepsilon)=h(\varepsilon) \int_{\Omega}\left(\lambda u_{\varepsilon}-\frac{k(\varepsilon)}{h(\varepsilon)}\right)
$$

and so, since $u_{\varepsilon} \rightarrow u_{0}>0$ and by $(H 4)$, it follows that $J(\varepsilon)>0$ for $\varepsilon$ sufficiently small. This completes the proof.

The following result gives us a bound of the optimal control, and it will be used to prove its uniqueness.

Lemma 4.2 Assume $(H 3)-(H 5)$. If $f \in \mathcal{C}$ is an optimal control, then

$$
f \leq T_{\lambda}
$$

where

$$
T_{\lambda}:=\inf \left\{t \in \mathbb{R}^{+}: \frac{k(t)}{h(t)}=\lambda \mathcal{K}\right\}, \quad \text { and } \quad \mathcal{K}:=\left(\frac{a_{M}}{e_{L}}\right)^{1 /(\beta-\alpha)}
$$

Remark $4.3 \quad$ a) $B y(H 4)$ and $(H 5)$, it follows that $T_{\lambda}>0$ and that $T_{\lambda} \rightarrow 0$ as $\lambda \downarrow 0$.

b) Theorem 4.1 and Lemma 4.2 are generalizations of Theorem 2.1 in [3], which has been proved in the case $m=1, h(t)=t$ and $k(t)=t^{2}$. 
Proof: Let $f \in L_{+}^{\infty}(\Omega)$ be. By $(H 5)$, there exists $t_{0}>0$ such that $k\left(t_{0}\right) / h\left(t_{0}\right)=\lambda \mathcal{K}$. We consider

$$
g:=\min \left\{f, t_{0}\right\}
$$

and we will prove that $J(g)>J(f)$, whence the result follows.

By definition, $g \leq f$ and then $u_{g} \geq u_{f}$. If $x_{0} \in \Omega$ is such that $f\left(x_{0}\right)=g\left(x_{0}\right)$ then

$$
\lambda u_{g}\left(x_{0}\right) h\left(g\left(x_{0}\right)\right)-k\left(g\left(x_{0}\right)\right) \geq \lambda u_{f}\left(x_{0}\right) h\left(f\left(x_{0}\right)\right)-k\left(f\left(x_{0}\right)\right) .
$$

On the other hand, if $f\left(x_{0}\right)>g\left(x_{0}\right)=t_{0}>0$, then by $(3.2)$

$$
\lambda u_{g}\left(x_{0}\right) h\left(g\left(x_{0}\right)\right)-k\left(g\left(x_{0}\right)\right) \leq \lambda \mathcal{K} h\left(t_{0}\right)-k\left(t_{0}\right)=0,
$$

and so by $(H 5)$, we get

$$
0 \geq \lambda u_{g}\left(x_{0}\right) \frac{h\left(g\left(x_{0}\right)\right)}{g\left(x_{0}\right)}-\frac{k\left(g\left(x_{0}\right)\right)}{g\left(x_{0}\right)}>\lambda u_{f}\left(x_{0}\right) \frac{h\left(f\left(x_{0}\right)\right)}{f\left(x_{0}\right)}-\frac{k\left(f\left(x_{0}\right)\right)}{f\left(x_{0}\right)} .
$$

Then,

$$
\begin{gathered}
J(g)=\int_{\{f=g\}} \lambda h(g) u_{g}-k(g)+\int_{\{f>g\}} \lambda h(g) u_{g}-k(g) \geq \int_{\{f=g\}} \lambda h(f) u_{f}-k(f)+ \\
+\int_{\{f>g\}}\left(\lambda \frac{h(g)}{g} u_{g}-\frac{k(g)}{g}\right) g>\int_{\{f=g\}} \lambda h(f) u_{f}-k(f)+\int_{\{f>g\}} \lambda h(f) u_{f}-k(f)=J(f) .
\end{gathered}
$$

For the uniqueness, we use the argument described in Section 6 in [3]. Firstly, we prove the next result.

Proposition 4.4 Let $J: \mathcal{D}:=\left\{f \in L^{\infty}(\Omega):(a-f) \in \mathcal{A}\right\} \subset L^{\infty}(\Omega) \mapsto \mathbb{R}$ be. Then $J$ is Fréchet continuously differentiable and

$$
J^{\prime}(f)(g)=\int_{\Omega}\left(\lambda h^{\prime}(f) u_{f}-\lambda u_{f}^{\alpha} P_{f}-k^{\prime}(f)\right) g, \quad \forall f \in \mathcal{D}, \forall g \in L^{\infty}(\Omega),
$$

where for any $f \in \mathcal{D}, P_{f} \in C_{0}^{1}(\bar{\Omega})$ is the unique solution of

$$
\left\{\begin{array}{rlr}
-\Delta P_{f}+M_{f}(x) P_{f} & =h(f) & \text { in } \Omega, \\
P_{f} & =0 & \text { on } \partial \Omega
\end{array}\right.
$$

being $M_{f}:=-\alpha(a-f) u_{f}^{\alpha-1}+\beta e u_{f}^{\beta-1}$. 
To prove this result, we need some previous ones. For $f \in \mathcal{D}$ and $g \in L^{\infty}(\Omega)$, let $\xi_{f, g}$ be the unique solution of

$$
\left\{\begin{aligned}
-\Delta \xi+M_{f}(x) \xi & =-g u_{f}^{\alpha} & & \text { in } \Omega, \\
\xi & =0 & & \text { on } \partial \Omega .
\end{aligned}\right.
$$

Observe that (4.2) and (4.3) have a unique solution because $\sigma_{1}\left(-\Delta+M_{f}\right)>0$ (see (3.10) and (3.11)) and Theorem 2.5.

Lemma 4.5 The map $f \in \mathcal{D} \mapsto P_{f} \in C_{0}^{1}(\bar{\Omega})$ is continuous.

Proof: Fixed $p>N$, we consider the map $\mathcal{G}: \mathcal{D} \times\left(C_{0}^{1}(\bar{\Omega}) \cap W^{2, p}(\Omega)\right) \mapsto L^{p}(\Omega)$ defined by

$$
\mathcal{G}(f, P)=-\Delta P+M_{f} P-h(f) .
$$

Observe that $\mathcal{G}$ is continuous. Indeed, the continuity of the map $f \mapsto M_{f} P$ follows with a similar argument to the one used in the proof of Theorem 3.3 to show that the map $D_{G} \mathcal{F}$ is continuous. On the other hand, it is clear that $\mathcal{G}\left(f, P_{f}\right)=0$. Given $\xi \in C_{0}^{1}(\bar{\Omega}) \cap W^{2, p}(\Omega)$ is easy to prove that $D_{2} \mathcal{G}\left(f, P_{f}\right) \xi=-\Delta \xi+M_{f} \xi$. Moreover, as in $(3.10), \sigma_{1}\left(-\Delta+M_{f}\right)>0$ and so $D_{2} \mathcal{G}\left(f, P_{f}\right)$ is non singular. The Implicit Function Theorem completes the proof.

The next result is due by Krasnoselskii, see [7], where we send for the definitions of the following concepts.

Lemma 4.6 Let $E$ be a Banach space ordered by a generating positive cone $P, F$ a Banach space and $T: E \mapsto F$. Assume that the Gâteaux derivative of $T$ with respect to $P$, denoted by $D_{G, P} T$, exists and it is continuous in a neighbourhood of $x_{0} \in E$. Then, the Fréchet derivative coincides with the Gâteaux derivative and $T$ is $C^{1}$ near $x_{0}$.

Recall that $P$ is generating if $E=P-P$. It is well known, see Proposition 1.7 in [1], that if $\operatorname{int}(P) \neq \emptyset$, then $P$ is generating.

Proof of Proposition 4.4: Firstly, we compute the Gâteaux derivative respect to the cone, denoted by $D_{G, P} J$. Let $g \in L_{+}^{\infty}(\Omega), f \in \mathcal{D}$ and $\varepsilon>0$ be such that $f+\varepsilon g \in \mathcal{D}$. Using Lemma 3.5 and (4.3)

$$
D_{G, P} J(f) g:=\lim _{\varepsilon \downarrow 0} \frac{J(f+\varepsilon g)-J(f)}{\varepsilon}=\int_{\Omega} \lambda \xi_{f, g} h(f)+\lambda h^{\prime}(f) u_{f} g-k^{\prime}(f) g .
$$


By the equation that satisfy $\xi_{f, g}$ y $P_{f}$ (see (4.3) and (4.2)), it follows that

$$
\int_{\Omega} h(f) \xi_{f, g}+\int_{\Omega} g u_{f}^{\alpha} P_{f}=0
$$

and so,

$$
D_{G, P} J(f)(g)=\int_{\Omega}\left(\lambda h^{\prime}(f) u_{f}-\lambda u_{f}^{\alpha} P_{f}-k^{\prime}(f)\right) g, \quad \forall g \in L_{+}^{\infty}(\Omega) .
$$

Let $f_{n} \rightarrow f \in \mathcal{D}$ be in $L^{\infty}$ and $g \in L^{\infty}(\Omega)$. Then, by Theorem 3.3 and Lemma 4.5 it follows

$$
\begin{gathered}
\sup _{\|g\|_{\infty} \leq 1}\left|D_{G, P} J\left(f_{n}\right)(g)-D_{G, P} J(f)(g)\right| \leq \\
\leq \sup _{\|g\|_{\infty} \leq 1} \int_{\Omega}\left|\lambda\left(h^{\prime}\left(f_{n}\right) u_{f_{n}}-h^{\prime}(f) u_{f}\right)-\lambda\left(u_{f_{n}}^{\alpha} P_{f_{n}}-u_{f}^{\alpha} P_{f}\right)-\left(k^{\prime}\left(f_{n}\right)-k^{\prime}(f)\right) g\right| \rightarrow 0 .
\end{gathered}
$$

and so, $D_{G, P} J$ is continuous. Applying Lemma 4.6, the Gâteaux derivative coincides with the Fréchet derivative and that the map is $C^{1}$.

The next result shows that some maps involved in (4.1) are Lipschitz continuous.

Lemma 4.7 Assume $(H 3)-(H 5)$. There exists $\Lambda>0$ such that for $0<\lambda<\Lambda$ the maps $f \in\left[0, T_{\lambda}\right] \mapsto u_{f}, P_{f}, u_{f}^{\alpha} P_{f} \in L^{\infty}(\Omega)$ are Lipschitz continuous.

Proof: Let $f, g \in\left[0, T_{\lambda}\right]$ be, by the monotony of the map $f \mapsto u_{f}$, it follows that

$$
0<u_{T_{\lambda}} \leq u_{f}, u_{g} \leq u_{0}
$$

for $\lambda$ such that $a-T_{\lambda}>0$, that is $\lambda<\lambda_{0}$ for some $\lambda_{0}$ (see Remark 4.3 a)). To the end of the proof we take $\lambda<\lambda_{0}$. By the Mean Value Theorem,

$$
\begin{gathered}
u_{f}^{\alpha}-u_{g}^{\alpha}=\alpha \xi^{\alpha-1}(f, g)\left(u_{f}-u_{g}\right), \quad u_{f}^{\beta}-u_{g}^{\beta}=\beta \eta^{\beta-1}(f, g)\left(u_{f}-u_{g}\right) \quad \text { with } \\
0<u_{T_{\lambda}} \leq \min \left\{u_{f}, u_{g}\right\} \leq \xi(f, g), \eta(f, g) \leq \max \left\{u_{f}, u_{g}\right\} \leq u_{0} .
\end{gathered}
$$

Let $w:=u_{f}-u_{g}$ be. Then, $w$ satisfies

$$
(-\Delta+N(f, g)) w=(g-f) u_{g}^{\alpha}, \quad \text { in } \Omega, \quad w=0 \quad \text { on } \partial \Omega
$$

where $N(f, g):=-\alpha(a-f) \xi^{\alpha-1}(f, g)+\beta e \eta^{\beta-1}(f, g)$. Using $f \geq 0$ and (4.4), it follows that

$$
N(f, g) \geq-\alpha a \xi^{\alpha-1}(f, g)+\beta e \eta^{\beta-1}(f, g) \geq-\alpha a u_{T_{\lambda}}^{\alpha-1}+e \beta u_{T_{\lambda}}^{\beta-1}
$$

It is not hard to show that as $\lambda \downarrow 0$

$$
\int_{\Omega}\left(-\alpha a u_{T_{\lambda}}^{\alpha-1}+e \beta u_{T_{\lambda}}^{\beta-1}\right) \varphi^{2} \rightarrow \int_{\Omega}\left(-\alpha a u_{0}^{\alpha-1}+e \beta u_{0}^{\beta-1}\right) \varphi^{2} \quad \forall \varphi \in H_{0}^{1}(\Omega),
$$


and so, by Proposition 2.3 we obtain that

$$
\sigma_{1}(-\Delta+N(f, g)) \geq \sigma_{1}\left(-\Delta-\alpha a u_{T_{\lambda}}^{\alpha-1}+e \beta u_{T_{\lambda}}^{\beta-1}\right) \rightarrow \sigma_{1}\left(-\Delta-\alpha a u_{0}^{\alpha-1}+e \beta u_{0}^{\beta-1}\right)>0
$$

as $\lambda \downarrow 0$. Hence, there exists $\lambda_{1}>0$ such that

$$
N(f, g) \geq-\alpha a u_{T_{\lambda_{1}}}^{\alpha-1}+e \beta u_{T_{\lambda_{1}}}^{\beta-1}
$$

and

$$
\sigma_{1}(-\Delta+N(f, g)) \geq \sigma_{1}\left(-\Delta-\alpha a u_{T_{\lambda_{1}}}^{\alpha-1}+e \beta u_{T_{\lambda_{1}}}^{\beta-1}\right)>0 .
$$

Then, by (4.5), (4.6) and Lemma 2.6, we have that $w \leq \psi_{1}$ where $\psi_{1}$ is the unique solution of

$$
\left\{\begin{aligned}
-\Delta u+\left(-\alpha a u_{T_{\lambda_{1}}}^{\alpha-1}+e \beta u_{T_{\lambda_{1}}}^{\beta-1}\right) u & =(g-f) u_{g}^{\alpha} & & \text { in } \Omega, \\
u & =0 & & \text { on } \partial \Omega .
\end{aligned}\right.
$$

Interchanging $f$ and $g$, we get that $-w \leq \psi_{2}$ where $\psi_{2}$ is the unique solution of (4.7) with second member $(f-g) u_{f}^{\alpha}$. Then, taking into account that $u_{f}$ possesses a priori bound independient of $f$ (see (3.2)) and Theorem 2.5, it follows that

$$
\left\|u_{f}-u_{g}\right\|_{\infty}=\|w\|_{\infty} \leq \max \left\{\left\|\psi_{1}\right\|_{\infty},\left\|\psi_{2}\right\|_{\infty}\right\} \leq \max \left\{\left\|\psi_{1}\right\|_{C^{1}(\bar{\Omega})},\left\|\psi_{2}\right\|_{C^{1}(\bar{\Omega})}\right\} \leq C\|f-g\|_{\infty}
$$

This shows that the map $f \mapsto u_{f}$ is Lipschitz.

Before proving the Lipschitz character of the map $f \in\left[0, T_{\lambda}\right] \mapsto P_{f}$, we see that

$$
P_{f} \leq \mathcal{P} \quad \text { in } \Omega
$$

where $\mathcal{P} \in C_{0}^{1}(\bar{\Omega})$, independient of $f$. Indeed, let $f \in\left[0, T_{\lambda}\right]$ be, then $M_{f} \geq-\alpha a u_{T_{\lambda}}^{\alpha-1}+\beta e u_{T_{\lambda}}^{\beta-1}$, and so, using again Lemma $2.6 \mathrm{~b}), P_{f} \leq \mathcal{P}$ where $\mathcal{P}$ is the unique solution of

$$
\left\{\begin{aligned}
-\Delta u+\left(-\alpha a u_{T_{\lambda_{1}}}^{\alpha-1}+e \beta u_{T_{\lambda_{1}}}^{\beta-1}\right) u & =T \text { in } \Omega, \\
u & =0 \text { on } \partial \Omega,
\end{aligned}\right.
$$

where $T:=\max _{f \in\left[0, T_{\lambda}\right]} \max _{x \in \bar{\Omega}} h(f(x))$. This implies (4.9).

We will prove now that the map is Lipschitz. Let $f, g \in\left[0, T_{\lambda}\right]$ and $z:=P_{f}-P_{g}$ be. Then $z$ satisfies

$$
-\Delta z+M_{f} z=T(f, g), \quad \text { in } \Omega, \quad z=0 \quad \text { on } \partial \Omega,
$$


where

$$
T(f, g)=h(f)-h(g)+P_{g}\left[\alpha(a-f)\left(u_{f}^{\alpha-1}-u_{g}^{\alpha-1}\right)-\beta e\left(u_{f}^{\beta-1}-u_{g}^{\beta-1}\right)\right]+\alpha(g-f) P_{g} u_{g}^{\alpha-1} .
$$

Applying again the Mean Value Theorem, we get

$$
\begin{gathered}
u_{f}^{\alpha-1}-u_{g}^{\alpha-1}=(\alpha-1) \xi^{\alpha-2}(f, g)\left(u_{f}-u_{g}\right), \quad u_{f}^{\beta-1}-u_{g}^{\beta-1}=(\beta-1) \eta^{\beta-2}(f, g)\left(u_{f}-u_{g}\right) \\
0<u_{T_{\lambda}} \leq \min \left\{u_{f}, u_{g}\right\} \leq \xi(f, g), \eta(f, g) \leq \max \left\{u_{f}, u_{g}\right\} \leq u_{0} .
\end{gathered}
$$

Hence,

$$
T(f, g)=h(f)-h(g)+P_{g}\left[\alpha(\alpha-1)(a-f) \xi^{\alpha-2}-\beta(\beta-1) e \eta^{\beta-2}\right]\left(u_{f}-u_{g}\right)+\alpha(g-f) P_{g} u_{g}^{\alpha-1}
$$

By a similar argument to the used in the proof of (4.8), we obtain

$$
\left\|P_{f}-P_{g}\right\|_{\infty}=\|z\|_{\infty} \leq C\|T(f, g)\|_{\infty}
$$

Since $\mathcal{P} \in C_{0}^{1}(\bar{\Omega})$, and using (3.3), (3.7), (4.9) and (4.10), we obtain

$$
\begin{aligned}
\left\|\alpha(f-g) P_{g} u_{g}^{\alpha-1}\right\|_{\infty} & \leq C\|f-g\|_{\infty}\left\|P_{g} u_{T_{\lambda_{1}}}^{\alpha-1}\right\|_{\infty} \\
& \leq C\|f-g\|_{\infty} k_{1}^{\alpha-1}\left\|\mathcal{P} d_{\Omega}^{\alpha-1}\right\|_{\infty} \\
& \leq C\|f-g\|_{\infty}\left\|d_{\Omega}^{\alpha}\right\|_{\infty}\|\mathcal{P}\|_{C^{1}(\bar{\Omega})} \\
& \leq C\|f-g\|_{\infty} \text { with } C \text { independient of } f \text { and } g .
\end{aligned}
$$

On the other hand, using (4.8), (4.9) and (4.10)

$$
\begin{aligned}
\left\|\alpha(\alpha-1)(a-f) P_{g} \xi^{\alpha-2}\left(u_{f}-u_{g}\right)\right\|_{\infty} & \leq C\left\|\mathcal{P} \xi^{\alpha-2}\left(u_{f}-u_{g}\right)\right\|_{\infty} \\
& \leq C\left\|\mathcal{P} \xi^{\alpha-2} \max \left\{\left|\psi_{1}\right|,\left|\psi_{2}\right|\right\}\right\|_{\infty} \\
& \leq C\left\|\mathcal{P} d_{\Omega}^{\alpha-2} \max \left\{\left|\psi_{1}\right|,\left|\psi_{2}\right|\right\}\right\|_{\infty} \\
& \leq C\|\mathcal{P}\|_{C^{1}(\bar{\Omega})}\left\|d_{\Omega}^{\alpha}\right\|_{\infty} \max \left\{\left\|\psi_{1}\right\|_{C^{1}(\bar{\Omega})},\left\|\psi_{2}\right\|_{C^{1}(\bar{\Omega})}\right\} \\
& \leq C\|f-g\|_{\infty}
\end{aligned}
$$

with $C$ independient of $f$ and $g$. Analogously it can be treated the term $-e \beta(\beta-1) P_{g} \eta^{\beta-2}\left(u_{f}-\right.$ $\left.u_{g}\right)$. Then, since $h$ is Lipschitz in $\left[0, T_{\lambda}\right]$ and by $(4.11)$, it follows that the map $f \mapsto P_{f}$ is Lipschitz. 
Optimal control for degenerate logistic equation

Let $f, g \in\left[0, T_{\lambda}\right]$ be, we have

$$
\left\|u_{f}^{\alpha} P_{f}-u_{g}^{\alpha} P_{g}\right\|_{\infty} \leq\left\|\left(u_{f}^{\alpha}-u_{g}^{\alpha}\right) P_{f}\right\|_{\infty}+\left\|u_{g}^{\alpha}\left(P_{f}-P_{g}\right)\right\|_{\infty}
$$

By the Mean Value Theorem,

$$
\left\|\left(u_{f}^{\alpha}-u_{g}^{\alpha}\right) P_{f}\right\|_{\infty}=\left\|\alpha \xi^{\alpha-1} P_{f}\left(u_{f}-u_{g}\right)\right\|_{\infty} \leq C\|\varphi\|_{C^{1}(\bar{\Omega})}\|f-g\|_{\infty} \leq C\|f-g\|_{\infty} .
$$

It is sufficient to take $\Lambda:=\min \left\{\lambda_{0}, \lambda_{1}\right\}$. This completes the proof.

Theorem 4.8 Assume $(H 3)-(H 5)$. Then, there exists $\Lambda_{0}>0$ such that if $\lambda<\Lambda_{0}$ there exists a unique optimal control.

Proof: Let $f \in \mathcal{C}$ be an optimal control, then by Lemma 4.2

$$
f \in I:=\left[0, T_{\lambda}\right]_{\infty}
$$

We take $\lambda<\Lambda$ (the constant obtained in Lemma 4.7) and sufficiently small $\lambda$ such that $I \subset \mathcal{C}$. In $I$, convex, the strictly concave character of $J$ is equivalent to the monotony of $J^{\prime}$. Hence, by (4.1), for $f, g \in I$, we have that

$$
\begin{gathered}
\left(J^{\prime}(f)-J^{\prime}(g)\right)(f-g)=\int_{\Omega}\left[\lambda\left(h^{\prime}(f) u_{f}-h^{\prime}(g) u_{g}\right)+\lambda\left(u_{g}^{\alpha} P_{g}-u_{f}^{\alpha} P_{f}\right)-\left(k^{\prime}(f)-k^{\prime}(g)\right)\right](f-g) \leq \\
\leq \int_{\Omega}\left(\lambda L-k_{0}\right)(f-g)^{2}<0,
\end{gathered}
$$

taking $\lambda<k_{0} / L:=\Lambda_{1}$, where $L$ the Lipschitz constant of the maps $h^{\prime}, f \mapsto u_{f}, f \mapsto P_{f}$ and $f \mapsto u_{f}^{\alpha} P_{f}$ (see Lemma 4.7).

\section{Regularity of the optimal control and optimality system}

In this section we consider the special case $h(t)=t$ and $k(t)=t^{2}$, which satisfy clearly $(H 4)$ and $(H 5)$. Moreover, in this case

$$
T_{\lambda}=\lambda \mathcal{K}
$$

The following result provides us of a caracterization of an optimal control. It follows as Theorem 3.1 in [10], using now our Lemma 3.5.

Lemma 5.1 Assume $f \in \mathcal{C}$ and (H3). If $f$ is an optimal control, then

$$
f=\frac{\lambda}{2} u_{f}\left(1-u_{f}^{\alpha-1} P_{f}\right)^{+}
$$


The next result says us that the optimal control is a Hölder continuous function when $\lambda$ is small and it lets us write the optimality system.

Proposition 5.2 Assume (H3). There exists $\Lambda_{1}$ such that if $\lambda \leq \Lambda_{1}$, then $P_{f} \leq u_{f}^{1-\alpha}$. So, if $f$ is an optimal control, we have that

$$
f=\frac{\lambda}{2} u_{f}\left(1-u_{f}^{\alpha-1} P_{f}\right)
$$

Proof: Let $f$ be an optimal control. For $\lambda<\lambda_{0}:=a_{L} / \mathcal{K}$, we have that $u_{f} \geq u_{\lambda \mathcal{K}}>0$. As in Lemma 4.7, it follows the existence of $\lambda_{1}$ such that there exists a unique positive solution $\psi$ of

$$
\begin{cases}-\Delta \psi+\left(-a \alpha u_{\lambda_{1} \mathcal{K}}^{\alpha-1}+\beta e u_{\lambda_{1} \mathcal{K}}^{\beta-1}\right) \psi=\mathcal{K} & \text { in } \Omega, \\ \psi=0 & \text { on } \partial \Omega .\end{cases}
$$

By Lemma 2.6 and (3.2), it follows that

$$
P_{f} \leq \lambda \psi \quad \text { for } \lambda \leq \lambda_{1}
$$

We define now

$$
\lambda_{2}:=\inf _{x \in \bar{\Omega}} \frac{u_{\lambda_{1} \mathcal{K}}^{1-\alpha}}{\psi} \leq \inf _{x \in \bar{\Omega}} \frac{u_{f}^{1-\alpha}}{\psi} .
$$

Observe that $\lambda_{2}>0$. Indeed, since $\psi$ and $u_{\lambda_{1} \mathcal{K}}$ are positive functions, it follows the existence of a constant $k>0$ such that

$$
\frac{u_{\lambda_{1} \mathcal{K}}^{1-\alpha}}{\psi}>k d_{\Omega}^{-\alpha}>0 .
$$

Taking $\Lambda_{1}:=\min \left\{\lambda_{0}, \lambda_{1}, \lambda_{2}\right\}$ and taking into account (5.2) and the definition of $\lambda_{2}$, it follows $P_{f} \leq u_{f}^{1-\alpha}$, and as a consequence of Lemma 5.1, we obtain (5.1).

The following result is an easy consequence of the previous result and it provides us with the optimality system.

Corollary 5.3 Assume (H3) and $\lambda \leq \Lambda_{1}$. Then any optimal control $f$ may be expressed as in (5.1), where the pair $\left(u_{f}, P_{f}\right):=(u, P)$ satisfies

$$
\begin{cases}-\Delta u=u^{\alpha}\left(a-\frac{\lambda}{2} u+\frac{\lambda}{2} u^{\alpha} P-e u^{\beta-\alpha}\right) & \text { in } \Omega \\ -\Delta P+\left(-\alpha a u^{\alpha-1}+\beta e u^{\beta-1}\right) P=\frac{\lambda}{2}\left(u-u^{\alpha} P(1+\alpha)+\alpha u^{2 \alpha-1} P^{2}\right) & \text { in } \Omega, \\ u=P=0 & \text { on } \partial \Omega,\end{cases}
$$

and $u>0$. 
Optimal control for degenerate logistic equation

Acknowledgments. M. Delgado and A. Suárez thank to CICYT of Spain (MAR98-0486) and J. A. Montero thanks to "Junta de Andalucía" (FQM116) and DGESIC (PB98-1343) by the partial financial support to the elaboration of this work.

\section{References}

[1] Amann H (1976) Fixed point equations and nonlinear eigenvalue problems in ordered Banach spaces. SIAM Review 18:620-709.

[2] Bertsch M, Rostamian R (1985) The principle of linearized stability for a class of degenerate diffusion equations. J. Diff. Eqns. 57:373-405.

[3] Cañada A, Gámez JL, Montero JA (1998) Study of an optimal control problem for diffusive nonlinear elliptic equations of logistic type. SIAM J. Control Optim. 36:1171-1189.

[4] Delgado M, Suárez A (2000) On the existence of dead cores for degenerate Lotka-Volterra models. Proc. Royal Society of Edin. 130 A:743-766.

[5] Gurtin ME, MacCamy RC (1977) On the diffusion of biological populations. Math. Biosci. $33: 35-49$

[6] Hernández J, Mancebo F, Vega de Prada JM On the linearization of some singular nonlinear elliptic problem and applications, to appear in Ann. Inst. H. Poincare Anal. Non-Linearie.

[7] Krasnoselskii MA (1964) Positive solutions of operator equations. Noordhoff, Groningen.

[8] Kufner A (1980) Weighted Sobolev Spaces. Text zur Mathematik, 31, Teubner, Leipzig.

[9] Leung AW (1995) Optimal harvesting-coefficient control of steady-state prey-predator diffusive Volterra-Lotka systems. Appl. Math. Optim. 31:219-241.

[10] Leung AW, Stojanovic S (1993) Optimal control for elliptic Volterra-Lotka type equations. J. Math. Anal. Appl. 173:603-619.

[11] Montero JA (2000) A uniqueness result for an optimal control problem on a diffusive elliptic Volterra-Lotka type Equation. J. Math. Anal. Appl. 243:13-31.

[12] Kavian O (1993) Introduction à la théorie des points critiques et applications aux problèmes elliptiques. Springer-Verlag, Paris. 